\title{
Real Time Emulation of Dynamic Tariff for Congestion Management in Distribution Networks
}

Rasmussen, Theis Bo; Wu, Qiuwei; Huang, Shaojun

Published in:

Proceedings of the 2016 Asian Conference on Energy, Power and Transportation Electrification

Publication date:

2016

Document Version

Peer reviewed version

Link back to DTU Orbit

Citation (APA):

Rasmussen, T. B., Wu, Q., \& Huang, S. (2016). Real Time Emulation of Dynamic Tariff for Congestion Management in Distribution Networks. In Proceedings of the 2016 Asian Conference on Energy, Power and Transportation Electrification IEEE.

\section{General rights}

Copyright and moral rights for the publications made accessible in the public portal are retained by the authors and/or other copyright owners and it is a condition of accessing publications that users recognise and abide by the legal requirements associated with these rights.

- Users may download and print one copy of any publication from the public portal for the purpose of private study or research.

- You may not further distribute the material or use it for any profit-making activity or commercial gain

- You may freely distribute the URL identifying the publication in the public portal 


\title{
Real Time Emulation of Dynamic Tariff for Congestion Management in Distribution Networks
}

\author{
Theis Bo Rasmussen, Qiuwei Wu, Shaojun Huang \\ Center for Electrical Power and Energy \\ Department of Electrical Engineering \\ Technical University of Denmark \\ Kgs. Lyngby, 2800 Denmark \\ (thras, qw, shuang)@elektro.dtu.dk
}

\begin{abstract}
This paper presents the real time evaluation of the dynamic tariff (DT) method for alleviating congestion in a distribution networks with high penetration of distributed energy resources (DERs). The DT method is implemented in a real time digital testing platform that emulates a real distribution network. The platform returns power system status that indicates the efficiency of the DT method in a real power system as a congestion management tool. Results show that the current formulation of the DT method through direct current optimal power flow (DCOPF) has some limitations as reactive power flows are ignored in the DCOPF. Reactive power flows causes overloaded transmission lines when the DT method allows consumption at network buses that meets active power transfer capability of transmission lines. Based on the real time emulation of the DT method, possible changes are suggested for the DT method that increases the efficiency of the method.
\end{abstract}

Index Terms-Active distribution network (ADN), congestion management, distributed energy resources (DERs), dynamic tariff (DT), real time emulation.

\section{INTRODUCTION}

The Danish government has an ambitious plan to become independent of fossil fuels by 2050 [1]. In the Danish government energy strategy of 2050, an electrification of heating and transportation is predicted. A key challenge for future distribution networks is to allow integration of distributed energy resources (DERs) such as heat pumps (HPs) and electric vehicles $(E V s)$ while maintaining secure operation of the distribution network [3].

A high penetration level of DERs in the distribution network will pose threats of congesting power system equipment [2]. In today's distribution networks, congestion management is mainly carried out by reinforcing the existing grid [4], which is very expensive and difficult as the future distribution of DERs is unpredictable. In the Ideal Grid for All (IDE4L) project, the concept of an active distribution network (ADN) that controls DERs to alleviate congestion is proposed [3]. In principle, congestion can be alleviated by increasing the costs of operating DERs in an hour where congestion is a risk. Increased prices change the optimal consumption schedule of DERs and the aggregators can distribute DER consumption to multiple hours [3].

978-1-5090-6173-0/16/\$31.00 (C)2016 IEEE
One method that utilizes price changes to alleviate congestion in distribution networks is called the Day-ahead Dynamic Tariff (DT) method [5]. Previously, the DT method has been tested in an offline testing environment to assure that DSO and aggregator calculated energy plans are equal when using quadratic programming optimization models [5]. Based on these tests, the DT method shows great potential as a congestion management method that can be used in future distribution networks. For further evaluation of the DT method, it is necessary to implement the method in a more realistic testing environment than previously used.

The goal of this paper is to evaluate the efficiency of the DT method in an online testing environment, created by connecting a real time digital simulator (RTDS) with optimization software through a communication server. In the online testing environment, a model of a real $10 \mathrm{kV}$ distribution network is implemented and simulated in real time, and all communication is performed across the communication server. Therefore the results presented in this paper can be used to evaluate the efficiency of the DT method implemented in an emulation of a real distribution network.

This paper gives an overview of how the real time digital testing platform is established and an evaluation of the DT method's efficiency in alleviating congestion while utilizing direct current optimal power flow (DCOPF) calculated DTs. The paper is organized as follows. The concept of the DT method is briefly described in Section II. The creation and overview of the real time digital testing platform is described in Section III. Simulation results of test cases in the real time digital testing platform are presented in Section IV. Section V, concludes the key findings of this paper.

\section{DAY-AHEAD DYNAMIC TARIFF}

In the DT method, the flexibility and price-sensitive nature of the DERs in the system is utilized to alleviate congestion. The DSO can change the DT at different load points in the distribution network which have congestion issues. The aggregators that control DERs experiences an increased tariff and by optimizing the operational cost, they can shift consumption of DERs to hours with less congestion. A key advantage of this method is that it is performed in a decentralized manner [6]. 
The decentralized structure of the DT method means that only the DSO holds information about the grid topology and rated values of power system equipment. The DSO calculates the required DTs to alleviate congestion by utilizing predicted data and a quadratic programming optimization model [6]. The algorithm flow of the DT method is shown in Fig. 1, which starts as the DSO and aggregators predict consumption data of base load devices as well as DERs and the hourly electricity prices [5].

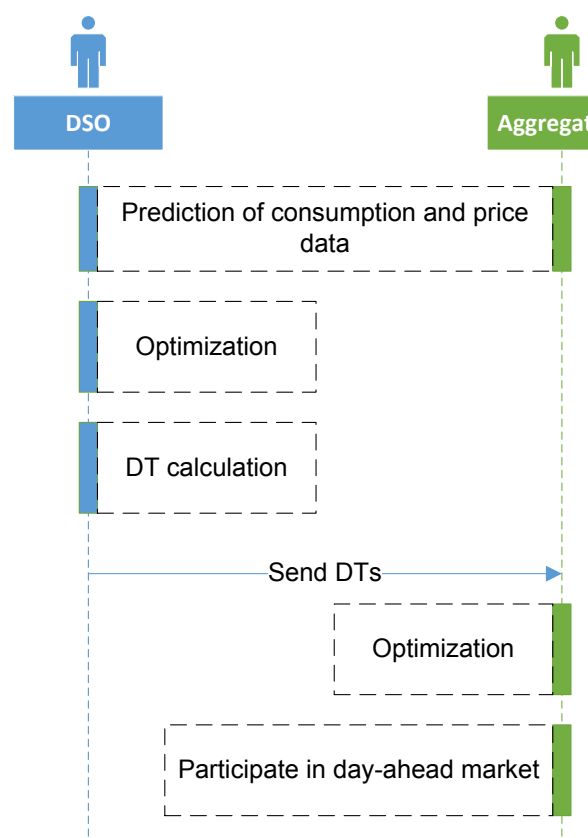

Fig. 1. Algorithm flow of DSO and aggregators in the DT method.

The DSO utilizes the predicted data, which is assumed stored in an accessible database, and topology data of the distribution network, to solve the quadratic programming model. The objective of the optimization model is to minimize the cost of operating the DERs in the network while satisfying the congestion constraints of the network. From the optimal solution, the DSO extracts the Lagrange multiplier of the congestion constraint for each bus in the network to calculate the DTs [5].

At the optimal solution, the Largrange multiplier can be characterized as the shadow price. The shadow price of a constraint describes the decrease of the minimized objective resulting from an expansion of the constraint right hand side value by a single unit [7]. From a congestion management perspective, the value of the Lagrange multiplier tells the DSO whether or not the optimal energy plans of the DERs in the system will cause a congestion if no corrective action is taken and how much the price of consuming electricity should be increased in hours and buses where congestion is a risk in order to avoid overloading the power system equipment. The calculated DTs are found using a DCOPF representation of the distribution network in which only active power transfer is considered in the optimization model [7].

After the DTs are calculated, they are communicated to the aggregators who calculate the optimal energy plans of subscribed DERs by including predicted electricity prices and DTs in their optimization models. Due to the quadratic optimization model of both the DSO and the aggregators and the inclusion of the DTs in the aggregator objective function, the congestion constraints are indirectly included in the aggregator optization model [5]. Afterwards, the aggregators will combine the optimal energy plans of all subscribed DERs and participate in the day-ahead electricity market. The detailed description of the mathematical models used in the DT method and the proof of convergence of DSO and aggregator optimal solutions can be found in [5].

\section{Real Time Digital Testing Platform}

The evaluation of the DT method in [5] utilizes a representation of the distribution network by considering power transfer capability of transmission lines as the only power system limit. In a real distribution network, more complicated parameters describe the status of the power system. These parameters include losses, voltage level at system buses as well as reactive power flows [8]. In order to further evaluate the efficiency of the DT method and whether the calculation of the DTs through a DCOPF representation of the distribution network can work in a real power system, the method is implemented in an emulation of a real power system referred to as a real time digital testing platform.

Based on the algorithm flow of the DT method shown in Fig. 1, the implementation of the DT method into a real time digital testing platform requires:

1) Two clients capable of using data from a database and find the optimal solution to a quadratic programming problem and

2) a communication channel between the DSO and the aggregator clients.

Additional requirements related to the emulation of a real distribution network are:

3) A model of a real distribution network implemented in a simulator capable of simulating power system dynamics to a certain degree of detail and

4) a way of communicating the hourly optimal energy plans calculated by the aggregators to the distribution network model to emulate hourly operation.

In order to satisfy these requirements, the authors identified appropriate software tools and made the simple network model shown in Fig.2. The optimization problems are solved at the DSO and aggregator clients by using the optimization software GAMS and the engineering toolbox Matlab. Matlab is used to load the predicted values from a database and format the data in GAMS readable files. When GAMS receives the required data, it will solve the optimization problem and for the DSO client calculate the DTs in the distribution network. The optimal solution is sent locally from GAMS to Matlab, which 
then connects to the Open Protocol Communication (OPC) server.

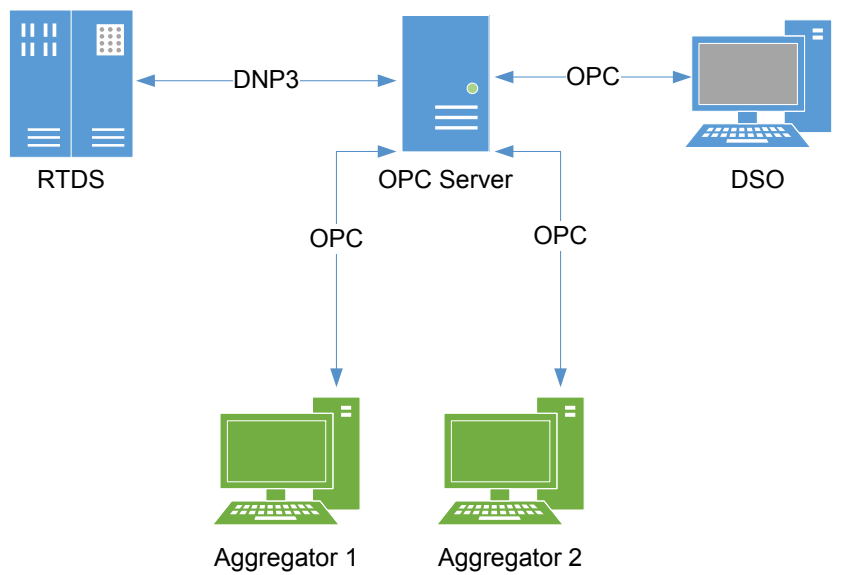

Fig. 2. Real time digital testing platform layout including communication links between clients and their respective communication protocols.

The task of the OPC server is to enable and allow communication of data between clients in the testing platform. An OPC server is a powerful tool capable of communicating across different protocols. The OPC standard, on which the server is based, is used in various industries to enable transfer of data between different devices and databases. As shown in Fig. 2, the OPC server can translate the OPC protocol data from the DSO and aggregators into the DNP3 protocol and vice versa, enabling two-way communication between all clients inside the real time digital testing platform [9].

In order to implement and simulate a real power system in real time, RTDS hardware and software is utilized as shown in Fig. 2. In power system research, RTDS is often used to simulate power system with new methods or algorithms to evaluate their efficiency before being applied to a real power system [10]-[12]. As such, the RTDS is an appropriate tool for calculating power system dynamics that affect the security of supply.

Through utilization of the real time testing platform, it is possible to emulate the DT method in a real power system. Instead of relying only upon the capabilities of the RTDS to show the efficiency of the DT method, the communication and calculation of DTs is emulated by introducing the DSO and aggregator clients as well as the OPC server. The platform is designed, but not limited to emulate the DT method in a real distribution network. Other market based methods for planning and managing the distribution network can be implemented in the platform shown in 2 . The real time digital testing platform therefore serves as a valuable tool for validating market based methods.

\section{Simulations And Results}

To test the efficiency of the DT method, DSO and aggregator clients are implemented as shown in Fig. 2, while the distribution network model implemented in the RTDS is one
$10 \mathrm{kV}$ feeder of the Bornholm distribution network [13]. The grid and DERs used in the case studies are described below, followed by a presentation of the test case and results.

\section{A. Distribution Network Model}

A single line diagram of the Bornholm $10 \mathrm{kV}$ feeder model is shown in Fig. 3. The grid is split in two radial feeders F1 and F2 which, in total, include 6 transmission lines labeled L1, L2, ..., L6, 8 transformers labeled T1, T2, ..., T6 and $610 \mathrm{kV}$ and $0.4 \mathrm{kV}$ buses labeled B1, B2, ..., B6. For buses $\mathrm{B} 2$ and $\mathrm{B} 5$, two transformers are connecting the $0.4 \mathrm{kV}$ bus to the $10 \mathrm{kV}$ grid due to high density of consumption, all transformers are assumed incapable of on-load tap changing and are implemented in the RTDS through detailed models from the RTDS software RSCAD. All lines are represented by pi equivalent circuits, transmission line $\mathrm{L} 1$ has a rated current of $0.241 \mathrm{kA}$ while all other transmission lines in the grid have rated current of $0.175 \mathrm{kA}$. The loads at the $0.4 \mathrm{kV}$ buses are residential modeled as dynamic loads which takes two signals from the OPC server as input, representing the total active and reactive power consumption, respectively, at each bus.



Fig. 3. Single line diagram representing part of the Bornholm distribution network divided into two feeders F1 and F2.

\section{B. Consumption and Price Data}

At each $0.4 \mathrm{kV}$ bus in the grid, a number of residents are located. The number of houses at each load point is found based on the yearly consumption at each bus obtained from the Danish DSO Østkraft. The peak load hour occurs at 19:00 and the peak base consumption can be seen in Table I. 
TABLE I

RESIDENTIAL BASE CONSUMPTION DATA.

\begin{tabular}{lcccccc}
\hline Bus & B1 & B2 & B3 & B4 & B5 & B6 \\
\hline Peak load $[k W]$ & 293 & 247 & 233 & 121 & 898 & 289 \\
No. Houses & 325 & 274 & 258 & 134 & 996 & 321 \\
PF. Lagging & 0.85 & 0.94 & 0.93 & 0.91 & 0.87 & 0.85 \\
\hline
\end{tabular}

If one of the houses connected to the grid is electrified, it is assumed that the owners have purchased both a HP and an EV. For all HPs in the system, a generic model with COP of 2.3 and a rated power of $6 \mathrm{~kW}$ is assumed. For each house, a surface area of 100 to $190 \mathrm{~m}^{2}$ is assumed randomly and the inside temperature set point is assumed to be $22^{\circ} \mathrm{C}$ with a deadband of $\pm 2^{\circ} \mathrm{C}$. For the EVs being implemented in the grid, one of three different currently available models are implemented based on the driving distance of each user [14] [16]. The EV characteristic data are shown in Table II and the driving distance of each $\mathrm{EV}$ owner is based on the results from a Danish driving pattern analysis described in [17].

TABLE II

ChARACTERISTIC DATA OF EVS

\begin{tabular}{lccc}
\hline Parameter & BMW i3 & VW e-Golf & Tesla Model S85 \\
\hline Capacity $[k W h]$ & 18.8 & 24.2 & 85 \\
Max charging $[k W]$ & 3.7 & 3.7 & 11 \\
Consumption $[W h / k m]$ & $129 \pm 10$ & $127 \pm 10$ & $236 \pm 20$ \\
Minimum SOC [\%] & 15 & 15 & 15 \\
Maximum SOC [\%] & 100 & 100 & 100 \\
Driving distance $[k m]$ & $<20$ & 20 to 50 & $>50$ \\
\hline
\end{tabular}

When calculating the optimal energy plan of the DERs, only the active power consumption is found due to the DCOPF formulation of the optimization problem. The reactive power at each load point is found by assuming a unity power factor of the EVs, a 0.84 lagging power factor for the HPs and a lagging power factor from 0.85 to 0.94 for the base consumption at the buses as shown in Table I.

The hourly electricity prices used in the case studies are generic data following a pattern where the price is lowest during the early morning and late evening.

\section{Test case}

Utilizing the real time digital testing platform, a test case was conducted to test the DT method in an emulation of a real power system. The test case is conducted with a $50 \%$ penetration of DERs in the network, meaning $50 \%$ of the houses in Table I have been electrified. In the test case, two scenarios were simulated to show the efficiency of the DT method compared to a situation where no congestion management control method is activated in the distribution network. The first scenario is with the DT method implemented to alleviate congestion in the distribution network. The second scenario is where the DT method is deactivated by setting all the DSO calculated DTs equal to zero before sending them to the aggregators through the OPC server as shown in Fig. 2

In this test case, 1392 EVs and HPs are implemented. By running the DT method to alleviate congestion, the DTs are found non-zero for feeders F1 and F2 for several hours as shown in Table III.

TABLE III

DYNAMIC TARIFFS IN DKK/1000 CALCULATED BY THE DT METHOD IN A TEST CASE WHERE 50\% OF THE HOUSES IN BORNHOLM FEEDER HAVE ELECTRIFIED BOTH HEATING AND TRANSPORTATION SERVICES.

\begin{tabular}{lcccccccc}
\hline Hour & $\mathbf{1}$ & $\mathbf{2}$ & $\mathbf{3}$ & $\mathbf{4}$ & $\mathbf{5}$ & $\mathbf{6}$ & $\mathbf{7}$ & $\mathbf{8}$ \\
\hline$D T_{F 1}$ & 1.9 & 4.9 & 5.8 & 11.0 & 0.7 & - & - & - \\
$D T_{F 2}$ & 72.7 & 74.5 & 74.4 & 80.3 & 70.3 & 63.6 & 57.7 & 49.5 \\
\hline Hour & $\mathbf{9}$ & $\mathbf{1 3}$ & $\mathbf{1 5}$ & $\mathbf{1 8}$ & $\mathbf{1 9}$ & $\mathbf{2 2}$ & $\mathbf{2 3}$ & $\mathbf{2 4}$ \\
\hline$D T_{F 1}$ & - & - & - & - & - & - & - & - \\
$D T_{F 2}$ & 13.3 & 4.4 & 1.8 & 87.2 & 1603.3 & 22.9 & 47.5 & 66.0 \\
\hline
\end{tabular}

For feeder F1 the DT is non-zero for hours 1 to 6 , while feeder F2 DTs equals zero in hours 10-12, 14, 16-17 and 2021. As explained in Section II, the DTs are only positive in hours where there is a risk of congestion. In hours where the base prices are high or the availability of DERs is limited, the optimal consumption of DERs is unable to exceed power system infrastructure limitations causing the DTs to equal zero. In I, the combined number of houses connected to feeder F2 is larger than the number of houses connected to F1, therefore, the occurrences where feeder $\mathrm{F} 2$ is experiencing congestion is higher than for feeder F1.

In order to see whether the DCOPF calculated DTs are adequate in order to alleviate congestion in the system, the line loading level is compared to the rated current of the transmission line in Fig. 4.

From the right plots of Fig. 4, showing the results from simulating the system without the DT method, it is clear that congestion management is required in the case of 50\% penetration of DERs. In this configuration, two lines in feeder F1 and one line in feeder F2 are overloaded in one or more hours of operation. The line L5 is the most overloaded and is, according to the simulation results, responsible for carrying $0.377 \mathrm{kA}$ which is more than twice the rated current of the transmission line.

The left plots in Fig. 4 show the results of simulating the system with the DT method. It shows that, for both feeders, the maximum loading level is decreased. However, even when increasing the price of consuming electricity in the hours with congestion, the lines L1, L2 and L5 are still overloaded. The cause of this operation is due to the reactive power being transferred in the transmission lines together with the active power.

As both the base consumption and the HPs have a lagging power factor, they absorb reactive power. This means that reactive power has to be supplied from somewhere in the grid, and as there are no reactive power generating units in the network, the reactive power is supplied by the external grid. 

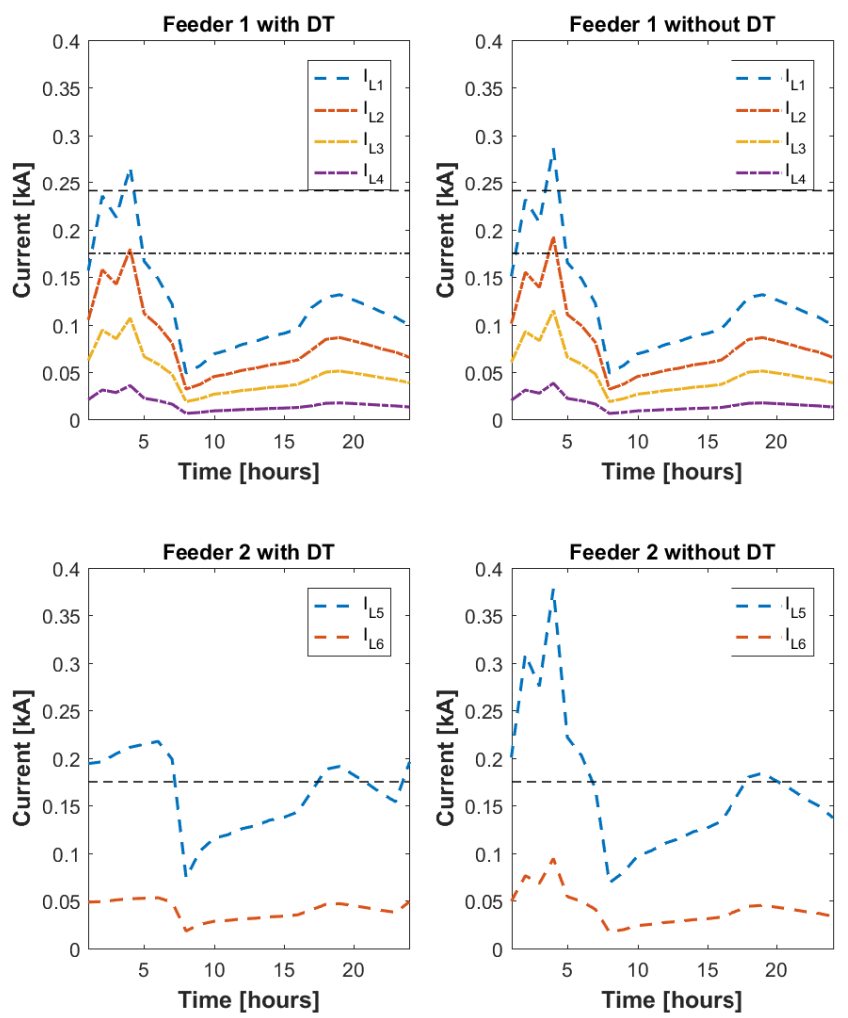

Fig. 4. Hourly line loading of feeder 1 and 2 where congestion management is necessary, compared to the rated current shown as black horizontal lines.

When the transmission lines has to transfer both active power at the rated level of the lines and additional reactive power, the absolute current increases beyond the rated current of the transmission line and induces greater losses in the system.

Another power system parameter that should be satisfied during operation of power systems is the voltage magnitude at the buses in the system. A general limit is that the voltage magnitude is allowed to vary by $10 \%$ from the rated voltage. In order to see which effect the DERs in the network and to further evaluate the DT method's efficiency, the bus voltage magnitude for the last $10 \mathrm{kV}$ bus of each feeder is shown in Fig. 5.

From the right plot in Fig. 5, the bus B6 voltage magnitude is $96 \mathrm{~V}$ lower in hour 4 in the scenario without the DT method compared to the scenario with the DT method. From this, it can be seen that the DT method indirectly affects the $10 \mathrm{kV}$ buses' voltage magnitude as the power consumption in this feeder is lowered due to the consumption price increase. By comparing the voltage magnitude at the different feeders with and without the DT method, it is seen that feeder F1 has the lowest voltage magnitude in the scenario without the DT method. In hour 4, the bus voltage magnitude of bus B4 in feeder F1 is equal to $9.575 \mathrm{kV}$. In the scenario with the DT method, it is also bus B4 that has the lowest voltage magnitude equal to $9.628 \mathrm{kV}$.

From the simulation results, all $10 \mathrm{kV}$ buses in the distribution network are operated well within the voltage limits. This
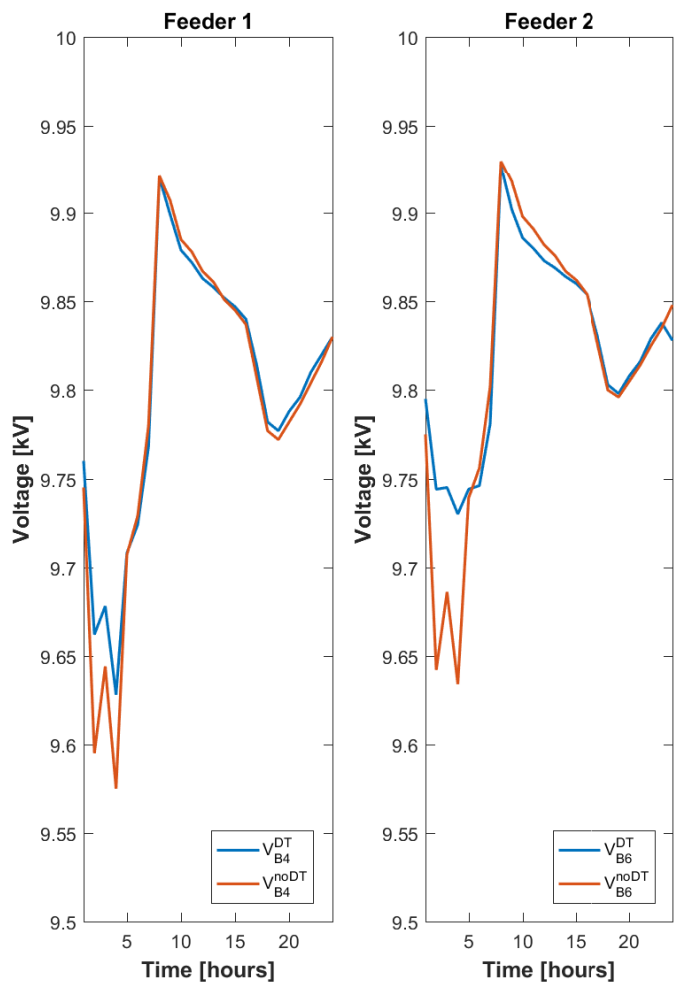

Fig. 5. Voltage magnitude at the $10 \mathrm{kV}$ bus furthest away from the external grid in each feeder.

applies to both scenarios with and without the DT method and as the voltage magnitudes of the buses, in the scenario with the DT method, are considerably larger than the limit of $9 \mathrm{kV}$, the DT method seems to have no problems with satisfying voltage limits even when using the DCOPF formulated optimization problem to calculate the DTs.

Results from testing the DT method in the real time digital testing platform show that the $0.4 \mathrm{kV}$ buses are likewise operated well within their operating limits. These results are not shown here due to space limitations.

\section{CONCLUSION}

From the evaluation and testing of the DT method performed in this paper, the efficiency of the DT method is affected by the calculation of DTs through a DCOPF representation of the distribution network. As power losses and reactive power flows in the network are ignored in calculating the DTs, the DT method is unable to completely alleviate congestion as lines are overloaded due to inductive currents transferring the reactive power in the network. Results from the real time digital testing platform show that voltage is not an issue when implementing the day-ahead dynamic tariff method in the distribution network used in this research study.

The efficiency of the method can be increased by utilizing ACOPF representation of the network instead of DCOPF in the optimization model, or by implementing reactive power 
control that can control load power factor to be at unity. If the DSO wishes to use a DCOPF formulation of the optimization model, the loading of transmission lines could be decreased by a certain percentage margin to allow reactive power flows as well as transmission losses. Further studies could be to investigate one of the presented solution to improve the efficiency of the method.

\section{REFERENCES}

[1] The Danish Government, "Energy strategy 2050," Technical report, Feb. 2011.

[2] S. Huang, Q. Wu, Z. Liu, A. H. Nielsen, "Review of congestion management methods for distribution networks with high penetration of distributed energy resources," in IEEE PES Innovative Smart Grid Technologies, Europe, Istanbul, Turkey, 2014, pp. 1-6.

[3] Tampere University of Technology, "Specification of active distribution network concept," Technical report, Ideal Grid for All (IDE4L), 2014.

[4] A. A. Sallam, O. P. Malik, "Fundamental concepts," in Electric Distribution Systems, 1st ed. Hoboken, NJ: John Wiley \& Sons Inc., 2011, ch. 1 , pp. 3-32.

[5] S. Huang, Q. Wu, Z. Liu, S. S. Oren, R. Li, "Distribution locational marginal pricing through quadratic programming for congestion management in distribution networks," in IEEE Transaction on Power Systems, Vol. 30(No. 4), pp. 2170-2178, 2015.

[6] R. Li, Q. Wu, S. S. Oren, "Distribution locational marginal pricing for optimal electric vehicle charging management," in IEEE Transaction on Power Systems, vol. 29(No. 1), pp. 203-2011. 2014

[7] A. J. Wood, B. F. wollenberg, G. B. Sheblé, Power Generation, Operation and Control, 3rd ed. Hoboken, NJ: John Wiley \& Sons Inc., 2014.

[8] P. Kundur, N. J. Balu, M. G. Lauby, Power System Stability and Control, 1st ed., Vol. 7, New York: McGraw-Hill NY, 1994.

[9] OPC Foundation (2016, June 22nd), "What is OPC?," [Website], Available: https://opcfoundation.org/about/what-is-opc/

[10] Y. Li, D. M. Vilathgamuwa, P. C. Loh, "Design, analysis, and realtime testing of a controller for multibus microgrid system," in IEEE Transaction on Power Systems, vol. 19(No. 5), pp. 1195-1204, 2004.

[11] W. Qiao, G. K. Venayagamoorthy, R. G. Harley, "Real-time implementation of a STATCOM on a wind farm equipped with doubly fed induction generators," in IEEE Transaction on Industry Applications, Vol. 45(No. 1), 2009

[12] X. Liu, A. Aichhorn, L. Liu, H. Li, "Coordinated control of distributed energy storage system with tap changer transformer for voltage rise mitigation under high photovoltaic penetration," in IEEE Transaction on Smart Grid, Vol. 3(No. 2), 2012.

[13] J. Østergaard, J. E. Nielsen, "The Bornholm power system an overview", PowerLabDK, Kgs. Lyngby, Denmark, 14th ed., 2011, Available: http://www.powerlab.dk/facilities/bornholmpowersystem.aspx

[14] BMW (2016, June 22nd), "Technical data," [Danish website], Available: http://www.bmw.dk/dk/da/newvehicles/i/i3/2013/showroom/technical_data.html

[15] VW (April 2014), "The new e-gold" [Danish publication]

[16] Tesla, "Model S," [Danish website], Available: http://www.teslamotors.com/da_DK/models

[17] Q. Wu, et al., "Driving pattern analysis for electric vehicle grid integration study", in IEEE PES Innovative Smart Grid Technologies, Europe, Gothenburg, Sweden, 2010, pp. 1-6. 